Revue d'histoire de l'Amérique française

REVUE D.HISTOIRE DE L'AMÉRIQUE FRANÇAISE

\title{
L’énergie nucléaire au Québec : débats politiques et conflits de représentations, 1963-1996
}

\section{Stéphane Savard}

Volume 69, numéro 3, hiver 2016

URI : https://id.erudit.org/iderudit/1035959ar

DOI : https://doi.org/10.7202/1035959ar

Aller au sommaire du numéro

Éditeur(s)

Institut d'histoire de l’Amérique française

ISSN

0035-2357 (imprimé)

1492-1383 (numérique)

Découvrir la revue

Citer cet article

Savard, S. (2016). L'énergie nucléaire au Québec : débats politiques et conflits de représentations, 1963-1996. Revue d'histoire de l'Amérique française, 69(3), 5-33. https://doi.org/10.7202/1035959ar
Résumé de l'article

À l'aide d'une approche centrée sur l'histoire de la culture politique, il est question dans ce texte d'une analyse, sur une période de près de quarante ans, de la place et du rôle joué par les représentations de l'énergie nucléaire au Québec. En étudiant les discours des responsables politiques québécois, des dirigeants d'Hydro-Québec et des nombreux acteurs sociaux qui prennent la parole pour s'opposer ou pour appuyer la production d'électricité par fission nucléaire, l'article met en lumière les conflits politiques et les luttes de représentations symboliques qui en découlent, de même que leurs conséquences dans le domaine énergétique. 


\title{
L'énergie nucléaire au Québec: débats politiques et conflits de représentations, I963-1996 ${ }^{1}$
}

\author{
STÉPHANe SAVARD \\ Département d'histoire et CRIDAQ \\ Université du Québec à Montréal
}

\begin{abstract}
RÉSUMÉ • À l'aide d'une approche centrée sur l'histoire de la culture politique, il est question dans ce texte d'une analyse, sur une période de près de quarante ans, de la place et du rôle joué par les représentations de l'énergie nucléaire au Québec. En étudiant les discours des responsables politiques québécois, des dirigeants d'Hydro-Québec et des nombreux acteurs sociaux qui prennent la parole pour s'opposer ou pour appuyer la production d'électricité par fission nucléaire, l'article met en lumière les conflits politiques et les luttes de représentations symboliques qui en découlent, de même que leurs conséquences dans le domaine énergétique.
\end{abstract}

ABSTRACT - By means of an approach grounded in the history of political culture, the article analyzes the role and importance of media and public images of nuclear power in Quebec over a period of nearly forty years. The paper studies the discourse of Quebec's political leaders, the directors of Hydro-Quebec and various other social actors who spoke in public either in support of or in opposition to the production of electricity by nuclear fission. In the process, the article sheds light on the political conflicts over nuclear power and the ensuing struggles over its symbolic representations. It also highlights the consequences of these debates for the future of the energy sector.

1. L'auteur remercie Antoine Brousseau-Desaulniers, Jean-François Beaudet et Carl Robichaud pour leur excellent travail de recherche en archives ainsi que les évaluateurs anonymes pour leurs commentaires forts pertinents. Enfin, il tient également à souligner l'appui financier du Fonds de recherche québécois sur la société et la culture (FRQSC). 
On est des hydrauliciens, mais on pense que le Québec et les Québécois devraient devenir des gens nucléaires.

Robert Boyd, 1977

Il est évident que la filière nucléaire, fortement contestée dans la plupart des pays du monde, n’est pas la mieux placée pour répondre aux critères du développement durable.

Alban D’Amours, 1996

$\mathbf{E}^{\mathrm{n}}$ n 2008, le gouvernement libéral de Jean Charest annonce la réfection de Gentilly-2, la seule centrale nucléaire encore active au Québec ${ }^{2}$. Quatre ans plus tard, le gouvernement péquiste de Pauline Marois annule le projet de réfection et annonce la fermeture de la centrale qui s'échelonnera en plusieurs étapes sur une quarantaine d'années ${ }^{3}$. Cette décision suscite aussitôt la controverse alors que, d'un côté, plusieurs acteurs économiques de la région du Centre-du-Québec dénoncent cette fermeture et que, de l'autre, des citoyens et groupes environnementalistes l'applaudissent ${ }^{4}$.

Cet exemple illustre bien à quel point l'énergie nucléaire peut être une importante source de conflits sociopolitiques entourant les choix énergétiques que devrait faire une société donnée. Ces conflits plongent souvent leurs racines dans l'histoire contemporaine, influencés par les décisions politiques et les choix technologiques en matière énergétique, de même que par le contexte socioéconomique dans lequel s'inscrit le développement de l'atome civil. Si l'historiographie occidentale a porté son regard sur ces enjeux liés au nucléaire, il s'avère pertinent de les reprendre dans le contexte énergétique et politique québécois qui est somme toute assez particulier. Ainsi, du fait de son immense potentiel en ressources hydroélectriques, le Québec a rapidement fait le choix de l'hydroélectricité comme principale filière de production d'électricité. Le débat entourant l'énergie atomique s'inscrit donc dans ce contexte particulier où la technologie nucléaire entre moins en concurrence avec les filières électriques du charbon, du pétrole ou du gaz naturel - comme c'est le cas en Ontario,

2. Jean-Robert Sansfaçon, "Une décision économique», éditorial, Le Devoir, 20 août 2008, A-6.

3. Michel Corbeil, "Une fermeture rapide de Gentilly-2 à "analyser” ", Le Soleil, 31 janvier 2013; La Presse canadienne, "Gentilly-2 cesse de produire de l'électricité », Le Devoir, 29 décembre 2012.

4. Ibid.; Marc Rochette, "Gentilly-2 : le jour des comptes est arrivé», Le Nouvelliste, 29 janvier 2013. 
en France ou encore aux États-Unis -, mais se voit plutôt comparée à la filière de l'hydroélectricité, une technologie fièrement maîtrisée par Hydro-Québec et de multiples ingénieurs et scientifiques québécois et francophones depuis la fin des années 1950 au moins ${ }^{5}$.

Les études en sciences humaines, sociales et économiques portant sur l'énergie nucléaire sont plutôt nombreuses, particulièrement depuis les années 1980 et 1990. En France et dans le monde anglo-saxon, plusieurs recherches portent sur les conditions d'implantation et sur le développement technologique et commercial de l'industrie nucléaire, sans toutefois faire abstraction de ses liens avec le pouvoir politique ${ }^{6}$. D'autres montrent l'importance qu'occupent l'énergie atomique et le développement de l'industrie nucléaire dans l'émergence de références identitaires propres à certaines sociétés, particulièrement en $\mathrm{URSS}^{7}$ et en France où le nucléaire civil et militaire est perçu comme une source de fierté nationale ${ }^{8}$. Enfin, certains chercheurs mettent l'accent sur les conflits entre les groupes de pression et les responsables politiques au sujet de l'utilisation ou non de l'énergie nucléaire, conflits parfois ancrés dans le domaine des valeurs et des représentations ${ }^{9}$.

Au Québec et au Canada, les études en sciences humaines et sociales en lien avec l'énergie nucléaire se concentrent surtout sur le développement de l'industrie et de la technologie ${ }^{10}$, sur l'évolution d'une politique

5. Stéphane Savard, Hydro-Québec et l'État québécois, 1944-2005 (Québec, Septentrion, 2013).

6. James M. Jasper, Nuclear Politics: Energy and the State in the United States, Sweden, and France (Princeton, Princeton University Press, 1990); Earl R. Kruschke et Byron M. Jackson, Nuclear Energy Policy: A Reference Handbook (Santa Barbara, ABC-Clio, 1990); Terence Price, Political Electricity: What Future for Nuclear Energy? (Oxford-New York, Oxford University Press, 1990).

7. Paul R. Josephson, Red Atom: Russia's Nuclear Power Program from Stalin to Today (Pittsburgh, University of Pittsburgh Press, 2000).

8. Gabrielle Hecht, The Radiance of France: Nuclear Power and National Identity after World War II (Cambridge, The MIT Press, 2009).

9. Riley E. Dunlap, Michael E. Kraft et Eugene A. Rosa, dir., Public Reactions to Nuclear Waste: Citizen's Views of Repository Siting (Durham et Londres, Duke University Press, 1993); Francis Fagnani et Alexandre Nicolon, dir., Nucléopolis : matériaux pour l'analyse d'une société nucléaire (Grenoble, Presses universitaires de Grenoble, 1979); Colette Guedeney et Gérard Mendel, L'angoisse atomique et les centrales nucléaires: contribution psychanalytique et sociopyschanalytique à l'étude d'un phénomène collectif (Paris, Payot, 1973); Alain Touraine et al., La prophétie anti-nucléaire (Paris, Seuil, 1980).

10. Que ce soit dans une perspective d'innovation technologique ou «d'échec technologique». Voir Robert Bothwell, Nucléus : l'histoire de l'Énergie atomique du Canada, Limitée (Montréal, Éditions Agence d'Arc, 1988); Yves Gingras et Mahdi Khelfaoui, «La centrale nucléaire Gentilly-1 : la trajectoire imprévisible d'une innovation technologique avortée", Revue d'histoire de l'Amérique française, 67, 1 (été 2013): 57-81; Mahdi Khelfaoui, Histoire de la centrale nucléaire de Gentilly-1, Mémoire de maîtrise (science, technologie et société), UQAM, 2013; Michel Trépanier, L’Aventure de la fusion nucléaire. La politique de la "Big Science» au Canada (Montréal, Boréal, 1995). 
nucléaire ${ }^{11}$ ou encore sur les luttes menées par les groupes environnementalistes dans les années $1970^{12}$. De rares travaux ont parfois analysé de manière parcellaire les conflits politiques au sujet de l'utilisation de l'énergie nucléaire ${ }^{13}$, mais jamais selon une approche diachronique dans la "longue durée " en ce qui a trait aux valeurs et représentations qui y sont rattachées. C'est cette lacune que nous souhaitons ici combler, alors que certains chercheurs ont récemment montré qu'il était possible d'étudier les enjeux énergétiques dans une perspective de culture politique centrée sur les conflits politiques et les luttes de représentations symboliques ${ }^{14}$.

En prenant comme point de départ cette problématique d'ensemble, le présent article désire ainsi analyser les discours des responsables politiques québécois, des dirigeants d'Hydro-Québec ainsi que des différents groupes de pression qui interviennent dans l'espace public et politique à l'égard du nucléaire entre le milieu des années 1960 et le milieu des années 1990. Ce faisant, il vise l'étude dans une perspective diachronique des valeurs et représentations que ces différents acteurs mettent de l'avant pour appuyer ou s'opposer à l'énergie atomique. En d'autres mots, cet article brosse le portrait d'une histoire politique des choix, valeurs et représentations qui sont rattachées à l'énergie nucléaire au Québec, une histoire sensible aux rapports de force et aux conflits qui surviennent entre les différents acteurs intéressés par la question.

L'analyse déployée dans ce texte repose sur un socle conceptuel qui mérite ici d'être brièvement exposé. Caractérisées par les valeurs, croyances,

11. R. Bothwell, Nucléus..., op. cit.; Brian Buckley, Canada's Early Nuclear Policy: Fate, Chance, and Character (Montréal-Kingston, McGill-Queen's University Press, 2000); Mahdi Khelfaoui, «Le nucléaire dans la stratégie énergétique du Québec, 1963-2012», Scientia Canadensis : revue canadienne d'histoire des sciences, des techniques et de la médecine, 37, 1-2 (2014): 105-132.

12. Ronald Babin, L'option nucléaire. Développement et contestation de l'énergie nucléaire au Canada et au Québec (Montréal, Boréal Express, 1984); Jean-Guy Vaillancourt, Mouvement écologiste, énergie et environnement. Essais d'écosociologie (Montréal, Albert Saint-Martin, 1982); Jean-Guy Vaillancourt, "Deux nouveaux mouvements sociaux québécois : le mouvement pour la paix et le mouvement vert», dans Gérard Daigle et Guy Rocher, dir., Le Québec en jeu. Comprendre les grands défis (Montréal, Presses de l'Université de Montréal, 1992), 791-807.

13. Voir R. Babin, L’option nucléaire..., op. cit.; M. Khelfaoui, «Le nucléaire...», loc. cit.; Étienne Lyrette, Évolution des controverses face à l'implantation d'une infrastructure nucléaire: les réfections des installations de stockage de déchets nucléaires de la centrale Gentilly-2 (1994 et 2004), Thèse de doctorat (Études urbaines), INRS et UQAM, 2010.

14. Caroline Desbiens, Power from the North: Territory, Identity, and the Culture of Hydroelectricity in Québec (Vancouver, UBC Press, 2013); Thibault Martin et Steven M. Hoffman, dir., Power Struggles: Hydro Development and First Nations in Manitoba and Quebec (Winnipeg, University of Manitoba Press, 2008); Stéphane Savard et Martin Pâquet, «Les enjeux énergétiques au Québec: conflits et représentations», Globe: Revue internationale d'études québécoises, 13, 2 (2010): 11-25 ; S. Savard, Hydro-Québec..., op. cit. 
images, opinions, attitudes ou symboles, les représentations sociales sont des interprétations ou des significations que plusieurs membres d'une société partagent au sujet d'une réalité donnée ${ }^{15}$. Lorsque cette réalité est complexe et abstraite et qu'elle relève du champ politique, ces représentations deviennent alors symboliques ${ }^{16}$ et identitaires, comme dans le cas qui nous intéresse ici. Véritables briques formant les murs de l'édifice de la société, ces représentations sont "imaginées ${ }^{17}$ » par les différents membres de la communauté, en particulier les responsables politiques. Puisque le "pouvoir représente», comme le mentionne l'anthropologue Marc Abélès, il devient alors possible d'étudier les représentations «imaginées" par les responsables politiques et les dirigeants d'une grande entreprise publique qui, tous deux, tentent de justifier leurs choix énergétiques devant la population. Par leurs interventions, ils visent la "mise en scène» de la collectivité dans un but de cohésion sociale et de recherche d'un consensus autour de ce qu'ils présentent comme étant le «bien commun ${ }^{18}$.

Mais ce n'est pas tout. Les représentations symboliques et identitaires "imaginées» voyagent à l'intérieur du champ sociopolitique ${ }^{19}$ qui, lui, renferme plusieurs autres acteurs qui participent au phénomène de prise de parole citoyenne, surtout à partir des années $1960^{20}$. Là, ces derniers tentent de faire valoir leurs intérêts et participent ainsi à des luttes de représentations, conflits qui détermineront en bout de piste quelles valeurs et représentations se montreront hégémoniques au sein de la société, lesquelles seront partagées par une majorité de citoyens et serviront donc à définir le «bien commun $»^{21}$. Dans le cas des enjeux énergéti-

15. Jean-Claude Abric, «La recherche du noyau central et de la zone muette des représentations sociales ", dans Jean-Claude Abric, dir., Méthodes d'étude des représentations sociales (Ramonville Saint-Agne, Éditions Érès, 2003), 59-80; Roger Chartier, Au bord de la falaise: l'histoire entre certitudes et inquiétude (Paris, Albin Michel, 1998), 78-79; Denise Jodelet, «Représentations sociales : un domaine en expansion », dans Denise Jodelet, dir., Les représentations sociales (Paris, Presses universitaires de France, 1997), 52-53.

16. Charles D. Elder et Roger W. Cobb, The Political Uses of Symbols (New York, Longman, 1983), 28-29.

17. Benedict Anderson, Imagined Communities : Reflections on the Origin and Spread of Nationalism (New York, Verso, 1991), 4-7.

18. Marc Abélès, «La mise en représentation du politique», dans Marc Abélès et Henri-Pierre Jeudy, dir., Anthropologie du politique (Paris, Armand Colin/Masson, 1997), 247-248 et 270.

19. Pierre Bourdieu, «La représentation politique : éléments pour une théorie du champ politique», Actes de la recherche en sciences sociales, 36-37 (février-mars 1981): 3-4 et 13.

20. Michel De Certeau, La prise de parole et autres écrits politiques (Paris, Seuil, 1994). Sur l'émergence de la prise de parole citoyenne au Québec dans les années 1960, voir Valérie Poirier et Stéphane Savard, "Le militantisme environnemental au Québec, ou comment l'environnement est devenu un enjeu politique», Bulletin d'histoire politique, 23, 2 (hiver 2015): 15-31.

21. R. Chartier, Au bord de la falaise..., op. cit., 78-81 et 100; Martin Pâquet et Marcel Martel, «L'effet miroir: la construction de la Communauté européenne et la rhétorique politique au Québec et au Canada, 1989-2000», Études internationales, XXXII, 3 (septembre 2001): 476-478. 
ques en lien avec le nucléaire, l'analyse de ces conflits de représentations est particulièrement intéressante afin de mieux comprendre les transformations de la place et du rôle joués par l'énergie atomique au Québec.

Divisée en cinq parties, l'analyse se concentre sur les périodes où se transforme la conception du rôle et de la place de l'énergie nucléaire au Québec, et où s'élaborent des consensus ou conflits entourant les valeurs et représentations qui y sont rattachées. La première partie (1963-1970) traite des débuts plutôt consensuels de l'énergie nucléaire au Québec, et des valeurs de modernité technologique qui y sont véhiculées. La deuxième section (1971-1976) met l'accent sur les conflits entre les responsables politiques du Parti libéral du Québec (PLQ) et ceux du Parti québécois (PQ) au sujet du développement de l'énergie nucléaire. La troisième partie analyse l'année 1977 comme un tournant dans les représentations du nucléaire au Québec, alors que le gouvernement Lévesque et plusieurs groupes de pression s'opposent à toute expansion de cette filière énergétique. Dans la quatrième section (1978-1983), il est question des débats sociopolitiques entourant le moratoire sur le nucléaire et de cette représentation de l'atome comme étant toujours la principale filière énergétique d'un futur plus ou moins lointain. Enfin, la dernière partie (1984 à 1996) analyse la poursuite du déclin du nucléaire, alors que cette forme d'énergie est désormais perçue comme étant problématique en raison de son inadéquation avec le concept de développement durable.

\section{LES DÉBUTS: L’APPEL DE LA MODERNITÉ TECHNOLOGIQUE, 1963- 1970}

Appelées les "Trente Glorieuses», les années d’après-guerre (1945-1973) sont caractérisées par une forte expansion économique. Au Canada, la période connaît en moyenne un taux de croissance du PIB réel de l'ordre de $5 \%$, ce qui engendre alors une substantielle augmentation des besoins énergétiques. Les décennies 1950 et 1960 ne sont d'ailleurs pas étrangères au développement tous azimuts de l'exploitation des puits de pétrole et de gaz naturel en Alberta, de même qu'à l'accélération des grands projets hydroélectriques au Québec et ailleurs dans les autres provinces canadiennes ${ }^{22}$.

L'augmentation constante de la demande en énergie, de même que le développement technologique accru depuis la Deuxième Guerre mondiale, encouragent la réalisation d'innovations technologiques dans le

22. Kenneth Norrie, Douglas Owram et J. C. Herbert Emery, A History of the Canadian Economy (Scarborough, Thomson/Nelson, 2002), 374-401. 
domaine énergétique un peu partout en Amérique du Nord. Au Canada, une des innovations technologiques les plus importantes dans ce domaine est la mise sur pied par Énergie atomique du Canada Ltée (EACL) d’un programme d'énergie nucléaire canadien en 1952. Dès 1955, EACL et Hydro-Ontario s'entendent pour expérimenter un prototype de réacteur nucléaire. En 1959, l'Ontario autorise la construction des premières centrales nucléaires au Canada : le réacteur Nuclear Power Demonstration à Rolphton et la centrale Douglas Point à Kinkardine. La province concrétise son virage nucléaire en 1964, alors qu'elle annonce la construction de la première centrale nucléaire commerciale d'envergure au pays: la centrale de Pickering ${ }^{23}$.

Dans le Québec du début des années 1960, qui connaît une forte croissance de l'économie, Hydro-Québec peine à répondre à la demande élevée d'énergie. Les prévisions de l'augmentation de la demande en électricité sont telles que, dans une conférence donnée en 1964, le commissaire JeanPaul Gignac affirme qu' "au rythme affolant où vont les choses », HydroQuébec «devra doubler sa production d'ici 10 ans (peut-être, éventuellement, tous les 6 ou 7 ans) [...] [et,] à compter de 1974, elle devra de

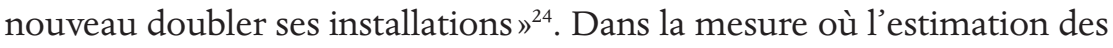
besoins futurs est fondée sur les données de la croissance économique du début des années 1960 et que les ressources hydrauliques rentables du Québec ne sont pas infinies, les dirigeants d'Hydro-Québec craignent alors un manque d'énergie hydroélectrique dans un avenir plus ou moins rapproché $^{25}$. Ce faisant, ils entament un processus de planification centré sur la diversification significative des moyens de production.

À une époque où s'avère particulièrement importante la foi envers le développement technologique pour résoudre les problèmes de l'humanité, les responsables politiques québécois et les dirigeants d'Hydro-Québec se tournent vers une augmentation des ressources allouées à la recherche scientifique. Non seulement la société d'État utilise-t-elle les nouvelles technologies pour améliorer le transport de l'électricité, notamment avec la création de l'Institut de recherche en électricité du Québec (IREQ), mais

23. R. Babin, L'option nucléaire..., op. cit., 32-41; R. Bothwell, Nucléus..., op. cit.; B. Buckley, Canada's Early Nuclear Policy..., op. cit.; Y. Gingras et M. Khelfaoui, «La centrale nucléaire Gentilly-1... », loc. cit., 59-65.

24. Archives d'Hydro-Québec [ci-après AHQ], H1, 505-000, 742, Hydro-Québec, Conférence de Jean-Paul Gignac (commissaire), 29 octobre 1964, 5. Ce discours est repris presque tel quel en 1968 par le ministre des Richesses naturelles Paul-E. Allard. Voir AHQ, H1, 505-000, 742, 642-110, Discours de l'Honorable Paul-E. Allard, ministre des Richesses naturelles, à l'occasion de la semaine de l'électricité, 12 février 1968, 11.

25. AHQ, H1, 505-000, 742, Hydro-Québec, Conférence de Jean-Paul Gignac..., op. cit., 5. 
elle fait également appel à ces dernières pour diversifier ses moyens de production et chercher à contrer un éventuel manque de ressources hydroélectriques. C'est ainsi qu'Hydro-Québec et le gouvernement Lesage se tournent vers l'énergie atomique qui est alors perçue, au Canada, en France ou aux États-Unis, comme «la technologie de l'avenir ${ }^{26}$ ».

À partir de 1963, le président d'Hydro-Québec, Jean-Claude Lessard, intègre le bureau des directeurs d'EACL et accepte que la société d'État participe à une étude sur la conception d'un nouveau type de réacteur nucléaire ${ }^{27}$. De plus, au moins depuis 1964, les dirigeants d'Hydro-Québec et les fonctionnaires du ministère des Richesses naturelles s'informent et réfléchissent quant aux possibles avantages commerciaux de l'électricité atomique $^{28}$. Il semble qu'Hydro-Québec commence à y voir plus clair dans ses études, alors que le commissaire Jean-Paul Gignac, en octobre 1964, laisse tomber:

Tout laisse croire que l'Hydro-Québec devra établir des centrales thermiques et nucléaires. [...] On estime que d'ici dix ans le coût des centrales nucléaires pourra diminuer et qu'elles deviendront peut-être plus économiques que les centrales thermiques conventionnelles, peut-être aussi économiques, tout compte fait, que les centrales hydroélectriques ${ }^{29}$.

Ces prévisions sont réitérées en 1966 par Jean-Claude Lessard qui brosse alors un portrait positif de la situation de l'énergie nucléaire civile au Canada et dans le monde entier ${ }^{30}$.

Diversification "obligée» des sources d'énergie et projections sur la rentabilité future des centrales nucléaires, telles sont les principales raisons qui expliquent le soudain intérêt d'Hydro-Québec et des responsables politiques envers l'énergie nucléaire dans les années 1960. À ces deux raisons peut s'ajouter une troisième: le "positivisme technologique» rattaché à l'énergie nucléaire ${ }^{31}$, que certains présentent comme un «tech-

26. Y. Gingras et M. Khelfaoui, «La centrale nucléaire Gentilly-1...», loc. cit., 60.

27. Ibid., 66-67.

28. Bibliothèque et Archives nationales du Québec Vieux-Montréal [ci-après BAnQ-M], P716: Fonds Michel Bélanger, Lettre de Douglas H. Fullerton à Michel Bélanger, [avril 1964], 2 p.

29. AHQ, H1, 505-000, 742, Hydro-Québec, Conférence de Jean-Paul Gignac..., op. cit., 9 et 11.

30. AHQ, H1, 505-000, 909, 642, Jean-Claude Lessard, Le Canada et l'énergie nucléaire, allocution prononcée par M. Jean-Claude Lessard, Président de l'Association canadienne d'énergie nucléaire, à Winnipeg, Manitoba, Canada, le 30 mai 1966, s.l., s.n., 1966, 2.

31. C'est l'amalgame entre l'énergie nucléaire, l'idée du progrès de l'humanité, les représentations de la modernité technologique et économique ainsi que l'enthousiasme débordant au sujet du programme de développement nucléaire, qui nous permet ici de souligner que le discours des acteurs étudiés représente une forme de "positivisme technologique». Au sujet de l'idée de progrès et de modernité en 
nological Enthousiasm ${ }^{32} »$. Ce dernier permet notamment aux responsables politiques et aux dirigeants de l'entreprise publique de rattacher le développement de l'atome civil à des représentations symboliques montrant un Québec "moderne» à la fine pointe de la technologie. Lors de la Conférence annuelle de la Canadian Nuclear Association en 1965, le premier ministre Lesage considère d'ailleurs que l'entrée dans "l'âge nucléaire" permettrait au Québec d'acquérir des connaissances technologiques et de contribuer à son développement économique:

Il ne servirait à rien de s'enfouir la tête dans le sable (je devrais peut-être dire "de la plonger dans nos abondantes réserves d'eau »). Nous sommes à l'âge nucléaire. Il faut le reconnaître et essayer de prendre les mesures nécessaires pour que les techniques nouvelles servent mieux les intérêts de l'humanité. En participant au développement de l'énergie nucléaire pour des œuvres de paix, nous aiderons à chasser les craintes et à faire avancer le monde sur la voie du progrès, ouverte il y a 61 ans par Rutherford, à Montréal ${ }^{33}$.

Dans les derniers mois de l'année 1965, le gouvernement Lesage autorise Hydro-Québec à construire, avec l'aide d'EACL, la première centrale nucléaire au Québec: Gentilly-1. De ce fait, suivant en cela plusieurs États nord-américains et occidentaux, les responsables politiques québécois se lancent ainsi avec enthousiasme sur la voie du nucléaire. Évident dans le discours de Jean Lesage de mai 1965, cet enthousiasme transparaît aussi dans l'esprit des hauts dirigeants hydro-québécois. Ainsi, dès 1966 devant l'Association canadienne d'énergie nucléaire, Jean-Claude Lessard souligne

qu'au seuil de l'ère nucléaire, l'Hydro-Québec espère pouvoir bientôt joindre sa contribution à la somme de connaissances et à l'expérience que vous avez déjà accumulées dans un domaine aussi riche de promesses pour l'avenir de l'humanité ${ }^{34}$.

Ce souhait se réalise officiellement le 12 novembre 1970 alors que la centrale Gentilly-1 entame ses essais de mise en service. Jean-Gilles Massé, ministre libéral des Richesses naturelles, en profite alors pour annoncer

lien avec le nucléaire, voir Y. Gingras et M. Khelfaoui, «La centrale nucléaire Gentilly-1...», loc. cit., 60; Yves Gingras, "Gentilly-2 - La fin de la saga nucléaire québécoise», Le Devoir, 15 octobre 2012.

32. Le chercheur James M. Jasper parle du «triumph of technological Enthusiasm » lorsqu'il analyse la perception de l'énergie nucléaire aux États-Unis de 1960 à 1973. Voir James M. Jasper, Nuclear Politics..., op. cit., 41-63.

33. Bibliothèque de l'Assemblée nationale du Québec [ci-après BAN], Jean Lesage, Discours du premier ministre du Québec, M. Jean Lesage, à la Conférence annuelle de la Canadian Nuclear Association, 11 mai 1965, 5.

34. AHQ, H1, 505-000, 909, 642, Jean-Claude Lessard, Le Canada et l'énergie nucléaire..., op. cit., 1. 
que ce "nouvel outil moderne d'exceptionnelle valeur» permet de «met[tre] l'atome à la disposition du Québec», un Québec qui «demeure [ainsi] à la fine pointe de la technique et de la science appliquée à notre époque moderne $»^{35}$. Le projet d'acquérir la technologie nucléaire et ainsi de faire entrer de plain-pied le Québec dans la modernité technologique se traduit même par la volonté de l'entreprise publique, dès 1966, de se lancer rapidement dans la construction d'une deuxième centrale nucléaire ${ }^{36}$. Il s'écoulera toutefois plusieurs années avant que l'État québécois n'annonce officiellement, en 1973, la mise en chantier de la centrale de Gentilly-2 (qui sera pleinement opérationnelle en 1983).

En bref, le choix de se tourner vers l'énergie nucléaire devient une manifestation d'un "positivisme technologique " centré sur l'idée de progrès technologique, de modernité économique et d'enthousiasme débordant. L'acquisition des connaissances pour faire fonctionner une centrale nucléaire, mais aussi et surtout la volonté bien arrêtée de contribuer à l'innovation mondiale en matière atomique, influencent les choix et orientations des responsables politiques et dirigeants de l'entreprise et les font imaginer ce que devrait être l’avenir énergétique du Québec.

\section{NUCLÉAIRE ET BAIE JAMES: DISCORDES AUTOUR DU PROGRAMME « MIXTE ॥, | 97 I - 1976}

La première moitié des années 1970 représente une intense période de débats politiques en ce qui concerne le choix des filières énergétiques que le Québec devrait privilégier. Les membres du gouvernement libéral de Robert Bourassa, et dans une certaine mesure les dirigeants d'HydroQuébec, continuent de s'appuyer fermement sur la filière de l'hydroélectricité en lançant en grande pompe le projet de la Baie James - celui du complexe La Grande - dès l'année 1971. Les Autochtones - Cris et Inuits - et plusieurs groupes environnementalistes et scientifiques critiquent le projet $^{37}$. Dans ce contexte, les valeurs et représentations associées à l'énergie nucléaire se fractionnent, contribuant à sectionner le fil du consensus qui semblait jusqu'alors relier entre eux les acteurs étudiés.

Les élus du PQ - guidés en cela par les idées et analyses de l'économiste Jacques Parizeau - poussent pour un plus grand investissement du Québec dans la filière atomique. Pour ces derniers, l'annonce de la mise en service

35. BNQ, Débats de l’Assemblée nationale du Québec [ci-après DANQ], 12 novembre 1970, 1503.

36. BAnQ-M, P716: Fonds Michel Bélanger, Oswald Mamo, «L’Hydro-Québec annonce la construction probable d'une autre centrale nucléaire dans la province», La Presse, le mardi 25 octobre 1966.

37. S. Savard, Hydro-Québec..., op. cit. 
de Gentilly-1 doit être perçue comme un premier pas vers l'installation de plusieurs autres centrales nucléaires ${ }^{38}$. Reléguant à l'arrière-plan le projet de la Baie James, ils cherchent plutôt à favoriser l'émergence d'une industrie nucléaire alors considérée comme une source de modernité pour le Québec. Cela permettrait, selon le député Lucien Lessard, d'éviter tout "retard technologique » qui obligerait, «lorsque nous en viendrons à créer de l'énergie nucléaire, [...] [à] tout simplement aller chercher ce qui s'est produit ou aller acheter nos produits en Ontario ${ }^{39}$. C'est ainsi que les députés péquistes considèrent que l'hydroélectricité ne serait pas l'unique source de modernité pour l'économie québécoise. L'atome permettrait aussi une industrialisation dite moderne. Dans un contexte où, depuis les années 1960, plusieurs chercheurs et grands commis de l'État s'intéressent notamment à la France en matière de planification économique ${ }^{40}$, n'est-il pas plausible de penser que Parizeau et ses collègues péquistes se soient alors inspirés de la politique énergétique de l'Hexagone qui emprunte à vive allure la voie du nucléaire, surtout après la crise pétrolière de $1973^{41}$ ?

Pour les responsables du gouvernement Bourassa, il est hors de question de mettre un frein à la production d'énergie hydroélectrique afin de privilégier celle d'une énergie nucléaire, qu'ils jugent plus coûteuse, plus polluante et aussi plus susceptible de rencontrer des problèmes ou des changements technologiques majeurs ${ }^{42}$. Ces craintes et images plutôt négatives associées au nucléaire représentent les premières manifestations, de responsables politiques québécois qui exercent le pouvoir, d'une certaine forme de nuance à l'égard du "positivisme technologique » qui avait influencé leur approche jusque-là. Cette position gouvernementale choque d'autant plus l'opposition péquiste que le contexte de contestations majeures du projet de la Baie James rend viable et plausible, selon eux, ce choix du nucléaire.

38. BNQ, DANQ, 12 novembre 1970, 1504.

39. BNQ, DANQ, 2 juillet 1970, 674. Sur la représentation du «retard», voir aussi René Lévesque, «La Baie (de) James en question... (4)» (mercredi le 6 mai 1971), dans Chroniques de René Lévesque (Montréal, Éditions Québec/Amérique, 1987), 176-177.

40. Voir notamment Jacques Benjamin, Planification et politique au Québec (Montréal, Presses de l'Université de Montréal, 1974); Michel Sarra-Bournet, Entretiens avec Louis Bernard (Montréal, Boréal, 2015), 35 .

41. A. Touraine et al., La prophétie anti-nucléaire, op. cit., 36; G. Hecht, The Radiance of France..., op. cit.

42. BNQ, DANQ, 9 juillet 1971, 3376-3377. Voir aussi BNQ, DANQ. Commission permanente des Richesses naturelles et des Terres et Forêts. Société d'aménagement de la baie James [ci-après Commission 1972], 16 mai 1972, B-1705; BNQ, DANQ. Commission permanente des richesses naturelles et des terres et forêts. Rapport des activités d'Hydro-Québec [ci-après Commission 1975], 4 juillet 1975, B-5477. 
En commission parlementaire au printemps 1972, Roland Giroux, président d'Hydro-Québec, reconnaît l'importance de "procéder graduellement à la construction de réacteurs nucléaires », préparant ainsi l'avenir par le développement d'une expertise et d'une industrie nucléaire québécoises $^{43}$. Pour les dirigeants, qui s'appuient sur les données de prévision de la demande d'électricité, les ressources hydroélectriques dans l'aprèsBaie James se feront de plus en plus rares et leur exploitation en sera dispendieuse. Ils entreprennent la planification d'un programme «mixte» au sein duquel l'hydroélectricité de la Baie James occuperait tout l'espace voulu, mais dans lequel il serait prévu d'intégrer graduellement la construction de plusieurs centrales nucléaires. Ce programme se transforme peu à peu, au fil des études régulièrement réajustées en fonction des prévisions sur l'augmentation de la demande en énergie. Il faut dire que ce programme "mixte» semble gagner en crédibilité à la suite de la crise énergétique de 1973-1974, alors que le prix du pétrole monte en flèche et que la construction de centrales nucléaires apparaît de prime abord plus avantageuse sur le plan économique ${ }^{44}$.

À tâtons, les dirigeants d'Hydro-Québec tentent ainsi de "préciser l'ampleur du programme à établir et à réaliser, pour obtenir la meilleure transition possible de l'hydraulique au nucléaire ${ }^{45}$ ». Dès 1974, ils jonglent avec l'idée d'installer une douzaine de centrales nucléaires dans les années $1990^{46}$. Les réflexions et calculs culminent en 1976 et 1977 avec l'élaboration d'un plan de construction qui prévoit l'implantation d'une trentaine de centrales nucléaires le long du Saint-Laurent jusqu’aux années 1990 ou 2000, sur le territoire de municipalités telles que Montmagny, Rivière-duLoup, Sainte-Croix ou Grondines ${ }^{47}$. «Notre but, dès lors», affirme Roland

43. Commission 1972, 16 mai, B-1707. Voir également ces prises de position antérieures chez certains dirigeants d'Hydro-Québec: Gaston Mathieu, «Réal Boucher: l'ambition a trop manqué aux gens de ma province », chronique Gros Plan, Entre Nous, mi-mars 1969, 6; Yvon De Guise, cité dans Daniel Dubeau, «L'Hydro-Québec construirait d’autres centrales nucléaires d'ici dix ans», Hydro-Presse, juillet 1971, 2.

44. M. Khelfaoui, "Le nucléaire...», loc. cit., 118.

45. Roland Giroux (président d'Hydro-Québec), devant la Commission permanente des Richesses naturelles le 3 juillet 1973, cité dans AHQ, H1, 1408, Résolutions: Hydro-Québec, À la recherche de sites thermiques et nucléaires, [1975], 1. Voir aussi "Gentilly 2: premier pas dans l'avenir», Hydro-Presse, fin octobre 1973, 6 .

46. BNQ, DANQ. Commission permanente des richesses naturelles et des terres et forêts. Rapport des activités de l'Hydro-Québec [ci-après Commission 1974], 4 juillet 1974, B-4835.

47. BNQ, DANQ. Commission permanente des Richesses naturelles et des Terres et Forêts. Programme d'investissements de l'Hydro-Québec [ci-après Commission 1976], 10 août 1976, B-3860; BNQ, DANQ. Commission permanente des richesses naturelles et des terres et forêts. Étude de la situation énergétique du Québec (février 1977) [ci-après Commission 1977], 9 février 1977, B-96; Commission 1977, 17 février, B-309; "La direction générale Génie se prononce sur l'avenir énergétique du Québec. L’an 2000, c’est demain», cahier spécial, Hydro-Presse, mi-février 1976, 7-10. Voir aussi R. Babin, L'option nucléaire..., op. cit., 63 ; Y. Gingras et M. 
Giroux, président d'Hydro-Québec, «est d'assurer une transition harmonieuse et sans heurt entre l'hydroélectricité et l'énergie nucléaire » ${ }^{48}$.

Au milieu des années 1970, les dirigeants d'Hydro-Québec sont donc convaincus de la nécessité de se tourner vers la production d'énergie nucléaire à grande échelle. Si l'idée d'un programme "mixte» à long terme ne semble pas poser problème aux yeux du gouvernement Bourassa, lui qui autorise la construction de Gentilly-2 en 1973, aucun des scénarios projetés n'est toutefois suffisamment avancé pour être approuvé à l’aube des élections de 1976. Quant aux élus du PQ, ils considèrent que le programme "mixte» ne répond pas à leurs attentes puisque ce dernier ne se mettrait pas en branle avant quelques années. Jacques-Yvan Morin revient en 1974 sur la notion de retard en affirmant que le plan d'Hydro-Québec d'installer une douzaine de centrales nucléaires dans les années 1990 arriverait trop tard, puisque

[...] le Québec sera [alors] complètement dépassé par ses voisins. À ce moment, il y aura certainement plusieurs dizaines de centrales en Ontario. Il y en aura 400 en Europe à la fin du siècle, pour l'an 2000. Le Québec sera le parent pauvre. Il devra importer sa technologie, il devra importer - oui, c'est cela la conséquence, il devra importer à peu près tout. Il devra même importer peut-être des spécialistes, et, comme par hasard peut-être qu'ils ne parleront pas français ${ }^{49}$.

Pour les péquistes, l'attente causée par la construction du projet de la Baie James est donc néfaste. Cette prise de position au sujet de l'atome subit toutefois un important virage dans les années qui suivent.

\section{LE TOURNANT: LE CHOC DES REPRÉSENTATIONS EN COMMISSION PARLEMENTAIRE, 1977}

Avec la victoire électorale du PQ en 1976, le conflit entourant l'énergie nucléaire ne se déroule plus uniquement dans l'arène politique, mais inclut désormais plusieurs acteurs tels que les groupes de pression environnementalistes et antinucléaires. Fondés pour la plupart au tout début des années 1970, ces derniers deviennent de plus en plus actifs à mesure que se cristallise le programme «mixte» hydroélectricité-nucléaire ${ }^{50}$. En

Khelfaoui, «La centrale nucléaire Gentilly-1...», loc. cit., 76; M. Khelfaoui, «Le nucléaire...», loc. cit., 118-120.

48. Commission 1976, 10 août, B-3860. Voir aussi R. Babin, L'option nucléaire..., op. cit., 63.

49. Commission 1974, 4 juillet, B-4835. Sur l'idée d'un grave retard, voir aussi son intervention en 1975 : Commission 1975, 3 juillet, B-5364-5365.

50. R. Babin, L'option nucléaire..., op. cit., 33 et 49. 
1976, ils profitent d'une ouverture de la «fenêtre des opportunités ${ }^{51}$ ", symbolisée par la volonté du nouveau gouvernement péquiste de doter le Québec d'une première véritable politique énergétique. Ils réclament alors la tenue d'un débat public sur l'énergie, ce qui permettrait ainsi à la question de l'énergie en général et du nucléaire en particulier de devenir un véritable enjeu de société. La réponse ne se fait pas attendre: le gouvernement Lévesque décide d'encadrer un mécanisme de consultation publique en invitant les individus et groupes intéressés à participer à la Commission parlementaire chargée d'étudier la situation énergétique du Québec prévue en février 1977.

Sur les 47 intervenants qui se présentent devant la Commission, 8 groupes ou individus interviennent contre l'énergie nucléaire, dont 5 groupes environnementalistes qui demandent l'imposition d'un moratoire sur la construction de nouvelles centrales nucléaires: le Comité de protection de l'environnement de Lotbinière, le Conseil québécois de l'environnement, le Regroupement pour la surveillance du nucléaire, la Société pour vaincre la pollution (SVP) et la Society to Overcome Pollution (STOP). Trois seulement soutiennent activement le développement de l'énergie nucléaire au Québec: Hydro-Québec, l’École Polytechnique de Montréal et la Fusion Energy Foundation. La polémique au sujet des choix et représentations entourant l'énergie nucléaire fait donc rage ${ }^{52}$.

Pour Hydro-Québec, «l’option nucléaire se présentera comme la seule forme d'énergie de relais fiable d'ici la fin du siècle». De $3 \%$ de toute l'électricité que la société d'État produira en 1985, la part de l'énergie nucléaire devrait augmenter à $33 \%$ en 2000 , alors qu'il ne resterait plus aucune ressource hydraulique à aménager à compter de $1993^{53}$. Comme le souligne Robert Boyd, président d'Hydro-Québec, en parlant des prévisions pour la décennie 1990 et les suivantes :

[...] je n'ai pas de boule de crystal, mais entre 1993 et 2000, il y a un problème. Il n'y aura plus d'hydraulique et même si nos maisons sont isolées, avec la laine comme cela à la grandeur et qu'il n'y a pas de fenêtre, tout est bouché, qu'on a tous des petites autos, il va quand même y avoir plus de maisons. Même si les gens n'ont qu'un poêle, la consommation d'énergie va avoir augmenté et on ne pourra pas fournir avec les rivières qu'on possède. C'est

51. Au sujet du concept de "fenêtre des opportunités», voir John W. Kingdon, Agendas, Alternatives, and Public Policies (Boston, Little, Brown and Co., 1984), 173-178.

52. Voir Commission 1977.

53. Pour la citation antérieure et ces informations, voir les discours des représentants d'Hydro-Québec dans Commission 1977, 15 février, B-170-171 et B-179-181. 
notre devoir de vous dire cela! On a l'air de dire: Malheureusement on n'a pas d'autre chose à vous offrir que du nucléaire. C'est ce qu'on a l'air de dire et c'est vrai que c'est cela qu'on vous dit. On est des hydrauliciens, mais on pense que le Québec et les Québécois devraient devenir des gens nucléaires ${ }^{54}$.

Alors que l'identité québécoise se veut rattachée aux prouesses d'HydroQuébec en matière d'hydroélectricité, le président de la société d'État souhaite poursuivre sur cette lancée et rendre "nucléaires» les Québécois. Cette référence identitaire s'inscrit dans un contexte international qui, faut-il le rappeler, joue en faveur du nucléaire, surtout en France qui est alors en voie de "nucléariser» sa production électrique.

Hydro-Québec considère également qu'il est primordial d'annoncer le plus rapidement possible la construction de futures centrales nucléaires afin de préserver le know-how québécois en la matière. Ainsi, pour les dirigeants de l'entreprise publique, l'expérience acquise avec les deux centrales (Gentilly-1 et Gentilly-2) n'est pas suffisante pour que les ingénieurs québécois puissent acquérir toutes les connaissances et techniques requises pour concevoir, construire et gérer des centrales nucléaires ${ }^{55}$. Il semble que ce soit sensiblement la même perception qui se dégage de la prise de position des porte-parole de l'École Polytechnique de Montréal. Ils rappellent ainsi que la formation des ingénieurs et techniciens dans le domaine du nucléaire prend du temps et qu'il faut dès maintenant poursuivre le développement québécois du nucléaire dans l'espoir d'employer près de 40000 travailleurs d'ici $2000^{56}$.

Cette perception qui présente l'énergie nucléaire comme étant inévitable dans un futur plus ou moins rapproché se voit quelque peu critiquée par Guy Joron, ministre délégué à l’Énergie. S’opère alors un spectaculaire changement d'orientation dans le discours péquiste. D’entrée de jeu, le ministre Joron précise les priorités de la future politique énergétique québécoise et des discussions qui suivront: la première priorité de son gouvernement est «d'établir un programme d'économie d'énergie» et "[d']éliminer, dans la mesure du possible, le gaspillage auquel on assiste»; la deuxième est de "rendre le Québec plus autarcique en matière énergétique $\aleph^{57}$. Par la suite, réagissant au discours des porte-parole de la société

54. Robert Boyd, dans Commission 1977, 15 février, B-184-185.

55. Voir les discours des représentants d'Hydro-Québec dans Commission 1977, 15 février, B-170-185.

56. Voir le discours de M. Amyot dans Commission 1977, 24 février, B-396.

57. Guy Joron, dans Commission 1977, 8 février, B-1-2. Précisons que ces objectifs sont une réponse à la Crise énergétique de 1973-1974. 
d'État, il souligne que l'utilisation du nucléaire n'est peut-être pas aussi inévitable que ces derniers le laissent entendre. Ainsi, tout en rappelant les fondements qui doivent servir à bâtir une véritable politique énergétique de l'État québécois, il suggère que les choix de société faits évacueront peut-être l'option nucléaire ${ }^{58}$.

Dans la réflexion entreprise par le gouvernement souverainiste qui planifie éventuellement un référendum sur la souveraineté, il est plausible de penser que l'énergie nucléaire est désormais perçue comme une énergie étrangère - dont la technologie est toujours maîtrisée par EACL, une société d’État canadienne qui chapeaute de manière étroite le travail d'exploitation des centrales nucléaires -, par opposition à l'hydroélectricité considérée comme "la seule forme d'énergie purement québécoise "59. De plus, il faut également noter que ce changement de cap survient alors que l'industrie nucléaire connaît une considérable augmentation de coûts. L'avantage de l'énergie nucléaire au début de la Crise du pétrole face à l'hydroélectricité s'amenuise considérablement quelques années plus tard en raison, entre autres, de l'inflation, ce qui fait gonfler significativement les coûts des grands projets énergétiques. Ce faisant, le gouvernement Lévesque remet en question, dès son arrivée au pouvoir, les bienfaits économiques et financiers d'un programme nucléaire ${ }^{60}$. Quoiqu'il en soit, la prise de position du ministre refroidit l'enthousiasme de plusieurs élus du PQ à l'égard du nucléaire, eux qui, jusqu'à l'aube des élections, défendaient encore bec et ongles un programme nucléaire plus costaud.

De leur côté, les groupes verts et antinucléaires craignent les "risques» du nucléaire et exigent au minimum un moratoire, sinon l'abandon pur et simple de la filière. Roger Lemay, porte-parole du Comité de protection de l'environnement de Lotbinière, met l'accent sur les déchets nucléaires, sur la contamination radioactive qui s'ensuit, sur les problèmes de rentabilité d'une centrale nucléaire ainsi que sur les conséquences néfastes pour «l'agriculture et les terres agricoles». Il affirme ainsi qu'en raison «de ces nombreuses et néfastes implications écologiques, sociales et économiques,

58. Guy Joron, dans Commission 1977, 15 février, B-183-184.

59. BAN, Guy Joron, Allocution du ministre délégué à l'Énergie, monsieur Guy Joron, lors du $28^{e}$ congrès provincial de la corporation des Maîtres électriciens : A-t-on tout dit sur l'impact de l'électricité au Québec? Jeudi 5 octobre 1978. Sherbrooke, Auberge des Gouverneurs, [s.1., s.n., 1978], 3-4; Gouvernement du Québec: Direction générale de l'énergie, La politique québécoise de l'énergie: assurer l'avenir (Québec, Éditeur officiel du Québec, 1978), 21.

60. R. Babin, L'option nucléaire..., op. cit., 161 et 164; M. Khelfaoui, «Le nucléaire...», loc. cit., 126; Commission 1977, 15 février, B-172; BNQ, DANQ. Commission permanente des Richesses naturelles et des Terres et Forêts. Étude des rapports des activités de l'Hydro-Québec et de la Société d'énergie de la baie James ainsi que tu nouveau règlement tarifaire (1), nº 191, 13 septembre 1977, B-5326. 
l'utilisation de l'énergie nucléaire comporte trop de risques et doit par conséquent être complètement abandonnée ${ }^{61}{ }^{1}$. Rappelant à quel point est importante la participation citoyenne dans le processus de prise de décision en matière d'enjeux énergétiques, il ajoute:

[1]'énergie est un bien, mais aussi un besoin collectif qui doit devenir la responsabilité de tous. Cette question, ce problème qu'est l'énergie, s'il demeure le monopole des seuls experts impliqués dans le développement et l'utilisation de l'énergie nucléaire, risque de nous entraîner vers des erreurs, des catastrophes dont les effets sont irréversibles ${ }^{62}$.

Pour Solange Vincent, porte-parole de la SVP, l'opposition au nucléaire ne représente pas simplement une prise de parole au sujet des choix énergétiques, mais aussi et surtout une contestation des valeurs véhiculées par les technocrates et les responsables politiques. Elle met l'accent sur la nécessité d'entreprendre "un réaménagement des valeurs prioritaires» de la société:

Donc, définissons quels pourraient être les principaux traits de ce nouveau visage de la société. Tout d'abord, nous croyons qu'une renégociation du "contrat» entre l'homme et le milieu naturel permettra aux deux parties de maximiser la qualité de leurs relations et d'en tirer des avantages insoupçonnés à long terme. Il faut donc cesser de planifier la destruction du milieu naturel, sinon nous risquons de prendre dramatiquement conscience des limites de la planète ${ }^{63}$.

Cette idée d'un choc des valeurs entre les opposants au nucléaire et ceux qui souhaitent son développement est reprise trois ans plus tard par Michel Jurdant, ardent environnementaliste et militant au sein du Conseil québécois de l'environnement. Ce dernier poursuit plus loin la réflexion de Roger Lemay et de Solange Vincent en précisant que la lutte des groupes environnementalistes se fait contre «l'électrosociété» et «l'électrotechnocratie» fondée sur une société de consommation ${ }^{64}$.

En plus de leur implication dans le processus de consultation citoyenne en commission parlementaire, les groupes verts et antinucléaires entreprennent des actions publiques pour réclamer un moratoire sur le nucléaire québécois et canadien et pour favoriser l'éducation de la population envers cette source d'énergie électrique considérée comme dange-

\footnotetext{
61. Roger Lemay, dans Commission 1977, 9 février, B-101 (voir aussi B-98-101).

62. Ibid., B-101.

63. Solange Vincent, dans Commission 1977, 17 février, B-311.

64. Michel Jurdant, dans Commission 1981, 25 février, B-2502.
} 
reuse pour les générations futures ${ }^{65}$. Ils mettent aussi sur pied une vaste «coalition verte» à l'été 1978. Cette dernière demande d'ailleurs à l'État québécois, dès l'année 1979, de lancer un véritable débat public sur l'énergie ${ }^{66}$. Comme le montrent si bien les travaux de Ronald Babin, ces groupes se transforment en un large mouvement social québécois et pancanadien qui préconise "un nouveau type de société avec une vision à long terme plutôt qu'à court terme ${ }^{67}$ ». Sur le plan des enjeux énergétiques, ils mettent donc l'accent sur une réduction de la consommation et sur des programmes d'économie d'énergie, rendant ainsi caduc, dans leur esprit, le développement d'une énergie nucléaire jugée dangereuse.

\section{POLITIQUE ÉNERGÉTIQUE ET MORATOIRE: L'ÉNERGIE NUCLÉAIRE JUGÉE RISQUÉE, 1978- 1983}

Comme les propos du ministre Guy Joron l’ont laissé présager pendant la commission parlementaire de 1977, le gouvernement Lévesque adopte finalement une position mitoyenne à l'égard de l'énergie nucléaire. La première véritable politique énergétique québécoise, annoncée en 1977 et dévoilée en 1978, met notamment l'accent sur le développement hydroélectrique et sur l'implantation d'un vaste programme d'économie d'énergie $^{68}$. Ce faisant, elle impose un moratoire sur la construction de nouvelles centrales nucléaires, repoussant ainsi le projet de construction de la centrale nucléaire de Gentilly-3, qui sera finalement abandonné en $1983^{69}$. Le moratoire de trois ans est reconduit en 1981, de même qu'en 1983 pour une période indéterminée cette fois ${ }^{70}$.

Cette décision du gouvernement Lévesque est influencée par une série de facteurs qui s'entremêlent et qui relèvent du contexte économique, politique et énergétique. Les deux facteurs précédemment expliqués sont toujours présents à la toute fin des années 1970: le développement du nucléaire québécois est dirigé en grande partie par une entreprise publique

65. Pour plus de détails, voir Stéphane Savard, «Les groupes verts et la question de l'énergie au Québec: émergence d'une prise de parole citoyenne, 1972-1997», dans Stéphane Savard et Jérôme Boivin, dir., De la représentation à la manifestation: groupes de pression et enjeux politiques au Québec, $19^{e}$ et $20^{e}$ siècles (Québec, Septentrion, 2014).

66. Les groupes verts et antinucléaires considèrent ainsi que le processus d'audiences publiques de la Commission de 1977, avec ses balises parlementaires, n'était pas un véritable débat public sur les enjeux énergétiques. Voir J.-G. Vaillancourt, «Deux nouveaux mouvements sociaux québécois..., loc. cit., 800.

67. R. Babin, L'option nucléaire..., op. cit., 158 (voir aussi 154-158).

68. Gouvernement du Québec, Direction générale de l'énergie, La politique québécoise de l'énergie: assurer l'avenir (Québec, Éditeur officiel du Québec, 1978).

69. M. Khelfaoui, «Le nucléaire...», loc. cit., 121.

70. Cette reconduction ne sera jamais sérieusement remise en question par les gouvernements subséquents. 
canadienne, et les prix du nucléaire connaissent d'importantes augmentations entre autres en raison de l'inflation galopante de la fin des années 1970. Soulignons également la crise économique de 1981-1982, la pire depuis la Grande Crise des années 1930, qui provoque une baisse considérable de la demande en électricité et un ajustement des prévisions hydro-québécoises ${ }^{71}$. À cela il faut ajouter les conséquences de l'accident nucléaire de Three Mile Island en 1979, aux États-Unis, qui créent une véritable onde de choc en Amérique du Nord et qui donnent des munitions, tout en venant grossir leur rang, de ceux et celles qui critiquent le choix du nucléaire ${ }^{72}$. Des représentations négatives de l'énergie nucléaire centrées sur la peur ou l'anxiété d'une catastrophe, jusqu'alors évoquées par les groupes environnementalistes sans grand succès, prennent de plus en plus de place au sein de la population en général ${ }^{73}$.

Malgré la décision gouvernementale d'établir un moratoire sur l'énergie nucléaire, les conflits de représentations se poursuivent au début des années 1980. Entre 1981 et 1983, le gouvernement Lévesque met sur pied deux commissions parlementaires sur les enjeux énergétiques et instaure un processus d'audiences publiques ${ }^{74}$. Si ce processus n'est pas considéré par les groupes verts comme un véritable débat public sur l'énergie, ce qui les amène ainsi en tant que membres du Front commun antinucléaire à boycotter la commission de 1981, il permet néanmoins de constater que la question du nucléaire ne semble pas complètement résolue. En effet, plusieurs intervenants trouvent important de faire entendre leur position

71. Commission 1977, 15 février, B-172; BNQ, DANQ. Commission permanente des Richesses naturelles et des Terres et Forêts. Étude des rapports des activités de l'Hydro-Québec et de la Société d'énergie de la baie James ainsi que du nouveau règlement tarifaire (1), 13 septembre 1977, B-5326. Voir aussi R. Babin, L'option nucléaire..., op. cit., 161 et 164; Y. Gingras et M. Khelfaoui, «La centrale nucléaire Gentilly-1...», loc. cit., 79.

72. Au pays de l'Oncle Sam, cet accident participe activement à une remise en question "about nuclear energy as a safe and viable element in future energy planning». Les risques associés à l'énergie nucléaire deviennent subitement réels, que ce soit sur le plan de la santé humaine avec les possibles fuites de radiation ou encore sur le plan de la destruction probable de l'environnement en cas de catastrophe. Voir E. R. Kruschke et B. M. Jackson, Nuclear Energy Policy..., op. cit., 5, 10 et 26; T. Price, Political Electricity..., op. cit., 10-11, 203 et 290. Pour les consequences de Three Mile Island au Québec, voir JeanPierre Rogel, «Événement. Le syndrôme de Three Mile Island», dans Face au nucléaire. Onze collaborateurs du magazine Québec Science (Québec, Québec Science éditeur, 1980), 300-301.

73. Comme l'explique T. Price pour le contexte américain, «[f]or many members of the Public any nuclear reactor is a potential Chernobyl or TMI [Three Miles Island]». Voir T. Price, Political Electricity..., op. cit., 290.

74. Il s'agit de la Commission permanente de l'énergie et des ressources de 1981 qui se penche sur le Plan d'équipement et de développement d'Hydro-Québec pour la décennie 1981-1990 ainsi que de la Commission permanente de l'énergie et des ressources de 1983 qui se penche sur l'étude des effets de la politique énergétique sur le développement économique du Québec. 
sur le sujet ${ }^{75}$. On y apprend ainsi que des membres du gouvernement Lévesque et de l'opposition jonglent toujours avec l'idée d'avoir un jour recours au nucléaire, mais à moindre échelle.

Ainsi, Yves Bérubé, ministre de l'Énergie en 1981, souligne que l'option nucléaire ne doit pas être écartée, compte tenu des limites théoriques du potentiel des rivières aménageables et en raison des coûts élevés du développement hydroélectrique des cours d'eau nordiques ${ }^{76}$. Il rappelle l'importance de conserver une expertise dans le domaine nucléaire, expertise qui permettra aux ingénieurs québécois de se plonger dans l'ère atomique en temps opportun ${ }^{77}$. Quant à Pierre-C. Fortier, porte-parole de l'opposition libérale en matière d'enjeux énergétiques, ses interactions avec les participants des commissions suggèrent que cet ancien président de Canatom, une entreprise ouvrant dans le domaine nucléaire, semble très favorable à une levée du moratoire et ainsi à la relance du nucléaire au Québec $^{78}$.

Même son de cloche du côté des dirigeants d'Hydro-Québec, alors qu'ils rappellent que les filières thermique classique et nucléaire sont les seules sources d'énergie pouvant servir à faire le pont entre l'épuisement des ressources hydrauliques et l'avènement des énergies nouvelles. Par contre, fait remarquable, ils reconnaissent, dès 1983, que le Québec n’aura pas besoin massivement de l'énergie nucléaire avant 2020 ou $2030^{79}$. Quant aux représentants de l'Ordre des ingénieurs du Québec, ils contestent néanmoins la position de l’État québécois en affirmant que le moratoire est «nuisible», car «il empêche toute discussion publique et éclairée ${ }^{80}$. Pour eux tout comme pour les porte-parole de l'École polytechnique, l'énergie nucléaire, combinée à l'énergie thermique classique, est la seule

75. La commission de 1981 a reçu 33 mémoires, dont 5 interviennent très clairement contre la fission nucléaire ou ne voient pas l'importance de cette source d'énergie dans le contexte actuel, alors que 6 autres se positionnent clairement en faveur de cette source d'énergie. Quant à la commission de 1983, elle a reçu 56 mémoires, dont 4 sont en faveur du maintien ou du développement de l'énergie nucléaire (fission nucléaire), alors que 4 militent plutôt pour une sortie du nucléaire.

76. Yves Bérubé, dans BNQ, DANQ. Commission permanente de l'énergie et des ressources. Plan d'équipement et de développement d'Hydro-Québec pour la décennie 1981-1990 (février-mars 1981) [ci-après Commission 1981], 24 février 1981, B-2338-2339.

77. Yves Bérubé, dans Commission 1981, 25 février, B-2433. Voir aussi son intervention du 26 février, B-2655-2657.

78. Commission 1981, 26 février, B-2655 ; Commission 1981, 2 mars, B-2764; BNQ, DANQ. Commission permanente de l'énergie et des ressources. Étude des effets de la politique énergétique sur le développement économique du Québec (mars - novembre 1983) [ci-après Commission 1983], 25 mars, B-73-75; Commission 1983, 21 septembre, B-7486.

79. En grande partie en raison de la révision à la baisse des prévisions de la demande en électricité, conséquence de la crise économique des années 1981 et 1982.

80. Commission 1981, 26 février, B-2651-2652. 
autre solution de rechange à l'hydroélectricité; les énergies nouvelles étant loin de pouvoir remplacer la quantité d'énergie produite par les centrales hydroélectriques ${ }^{81}$. Pour cette raison, l'Ordre des ingénieurs du Québec est persuadé, en 1981 comme en 1983, que le Québec doit investir dans la recherche sur le nucléaire, particulièrement dans celle sur la fusion nucléaire, à son tour perçue comme l'énergie de l'avenir ${ }^{82}$.

Qu'ils soient pour la relance du nucléaire ou encore pour le maintien du moratoire (sans l'abandon complet de la production d'énergie atomique), tous ces intervenants évoquent directement ou indirectement des représentations symboliques communes: celles d'une énergie nucléaire perçue comme étant l'énergie de l'avenir à laquelle les Québécois ne peuvent renoncer. En continuité avec les périodes antérieures, ces représentations s'avèrent toutefois davantage réalistes qu'idéalistes: l'enthousiasme face à la technologie qui accompagnait l'énergie nucléaire dans les années 1960 et au début des années 1970 a laissé place à un certain pragmatisme. Ainsi, parce que les responsables politiques et les dirigeants d'Hydro-Québec sont persuadés que seul le nucléaire pourra remplacer l'hydroélectricité à moyen et à long terme, ils considèrent encore que le Québec ne peut se passer de l'atome. S'ils se montrent désormais plus sensibles aux risques inhérents - et désormais concrets - reliés à cette filière énergétique, ils ne peuvent donc concevoir de mettre au rancart toute production d'énergie nucléaire.

À l'opposé des orientations énergétiques et des représentations symboliques déployées par ces acteurs se trouvent les choix et représentations des groupes environnementalistes et antinucléaires. En effet, ils mettent l'accent sur deux principales représentations pour exiger le maintien du moratoire, voire pour demander la fermeture de Gentilly-2:

1) la technologie du nucléaire est beaucoup trop risquée car elle n'est pas encore totalement maîtrisée, ce qui engendre donc des risques non négligeables de catastrophes ${ }^{83}$; et

81. Commission 1981, 25 février, B-2430 et B-2436; Commission 1981, 26 février, B-2651-2652; Commission 1983, 25 mars, B-74-75.

82. À ce sujet, l'Ordre des ingénieurs se réjouit du fait que le projet Tokamak à Varennes verra bientôt le jour. Avec ce projet, l'Ordre est persuadé que le Québec pourrait devenir un chef de file mondial dans le domaine de la fusion nucléaire, perpétuant ainsi les représentations de la modernité technologique et scientifique du Québec grâce aux recherches et à l'utilisation de l'énergie nucléaire. Voir Commission 1981, 26 février, B-2652; Commission 1983, 25 mars, B-71 et B-77.

83. Il s'agit du point de vue conjoint du Regroupement pour la surveillance du nucléaire et de l'Alliance-Tournesol, de même que des discours de la CSN et de la FTQ. Voir Commission 1981, 25 février, 2 mars et 3 mars. 
2) le Québec n'a pas besoin du nucléaire et n'en aura jamais besoin ${ }^{84}$.

Certains groupes comme le Regroupement pour la surveillance du nucléaire et l'Alliance Tournesol affirment même que toute implication du Québec dans l'industrie nucléaire ne fait que favoriser la «sale besogne d'aider la prolifération de l'armement nucléaire dans d'autres pays, dont des dictatures militaires comme celles de l'Argentine, de la Corée du Sud et du Pakistan ${ }^{85}$ ». Reprenant les arguments de maintien de l'expertise nucléaire à leur compte, mais d'une manière totalement inversée, ils soulignent d'ailleurs que

[p]our maintenir une expertise minimale dans le secteur nucléaire, objectif poursuivi par le gouvernement du Québec, au lieu de faire fonctionner Gentilly-2, Hydro-Québec devrait plutôt procéder au démantèlement de Gentilly-1 et ce, avec du financement venant d'Ottawa ${ }^{86}$.

L'expertise scientifique tant recherchée par l'État québécois et HydroQuébec se trouverait donc, à leurs yeux, dans le démantèlement des centrales nucléaires.

Dans les premières années de la décennie 1980, si bon nombre d'intervenants de divers horizons acceptent le moratoire sur la production d'électricité par fission nucléaire, plusieurs sont toutefois persuadés que cette forme d'énergie représente toujours la seule véritable solution à l'épuisement prévu des ressources hydroélectriques. Pour cette raison, un relatif consensus semble avoir émergé chez les responsables politiques : il faut exploiter la centrale Gentilly-2 afin que le Québec conserve une expertise dans le domaine nucléaire. Ce consensus tire toutefois à sa fin.

\section{L'INCOMPATIBILITÉ ENTRE LE NUCLÉAIRE ET LES PRINCIPES DE DÉVELOPPEMENT DURABLE, 1984-1996}

Entre 1984 et 1990, peu de débats à l'Assemblée nationale et en commissions parlementaires concernent l'énergie nucléaire. Depuis la crise économique de 1981-1982 et l'accumulation d'énergie électrique excédentaire, le champ de bataille se déplace plutôt vers le projet d'exportation massive d'électricité qui se déploie pleinement en 1985 avec l'arrivée du

84. Il s'agit entre autres du point de vue conjoint du Regroupement pour la surveillance du nucléaire et de l'Alliance-Tournesol. Voir Commission 1981, 2 mars; Commission 1983, 22 septembre.

85. Commission 1981, 2 mars, B-2763-2764. Ils rejoignent ainsi les arguments évoqués par les groupes environnementalistes américains et occidentaux depuis les années 1960 et 1970 au sujet des liens possibles entre le développement du nucléaire civil et celui du nucléaire militaire. Voir T. Price, Political Electricity..., op. cit., 175.

86. Jacques Boucher, dans Commission 1983, 22 septembre, B-7568. 
nouveau gouvernement Bourassa. Le premier ministre a la ferme intention de devancer la construction de centrales hydroélectriques afin d'éviter le " gaspillage» de l'eau et de faire du Québec un "Alberta de l'Est ${ }^{87}$ ». C'est ainsi qu'à la fin des années 1980, l’attention d'Hydro-Québec et du gouvernement Bourassa se tourne de nouveau vers le Nord québécois et ses ressources hydroélectriques non exploitées.

L'annonce du projet Grande-Baleine en 1989 sonne l'alerte parmi plusieurs groupes de pression qui dénoncent ses conséquences néfastes sur les écosystèmes nordiques et les populations autochtones et inuites. Devant le mécontentement populaire, le gouvernement Bourassa met sur pied une consultation générale sur la situation et les perspectives de l'énergie électrique au Québec. Lors de cette Commission parlementaire de 1990 qui étudie la position de 66 intervenants, un débat prend forme entre, d'une part, le gouvernement Bourassa, Hydro-Québec et plusieurs acteurs économiques et, d'autre part, les opposants au projet GrandeBaleine - surtout les groupes environnementalistes et autochtones ${ }^{88}$. Alors que le gouvernement Bourassa tente de justifier le projet Grande-Baleine, l'énergie nucléaire lui sert de repoussoir pour mieux illustrer les options énergétiques qui s’offrent aux Québécois. Ainsi, dans son discours d'ouverture, la ministre Lise Bacon met l'accent sur «les impacts réels ou appréhendés» de l'énergie atomique et se réjouit que «[n]ous n’utilisons que marginalement la filière nucléaire, ce qui nous permet d'éviter les risques [environnementaux et autres] attribués à cette forme d'énergie ». Cela lui permet de mieux faire valoir le projet Grande-Baleine et la production d'énergie hydroélectrique "propre» qui a permis au Québec de produire "per capita moitié moins de gaz carbonique que la moyenne canadienne ${ }^{89}$. De la même façon, dans un contexte de préoccupation grandissante au sujet de la lutte contre les gaz à effet de serre, Hydro-Québec rappelle les coûts financiers et environnementaux du nucléaire afin de mettre l'accent sur les avantages de l'hydroélectricité ${ }^{00}$. Cette position est également reprise par certains acteurs économiques tels que la Chambre

87. Robert Bourassa, Deux fois la Baie James (Montréal, Éditions La Presse, 1981), 55-56; Robert Bourassa, L'Énergie du Nord: la force du Québec (Montréal, Québec/Amérique, 1985), 20 et 100-104.

88. Pour une analyse plus fine des débats entourant Grande-Baleine, voir S. Savard, "Les groupes verts...", loc. cit., 123-130.

89. Lise Bacon, dans BNQ, DANQ, Commission permanente de l'économie et du travail. Consultation générale sur la situation et les perspectives de l'énergie électrique au Québec [ci-après Commission 1990], 8 mai 1990, CET-1506. Le terme "propre» n’est pas utilisé par Lise Bacon pendant la commission de 1990, mais par Robert Bourassa, moins d'un an plus tard, dans un contexte de débats entourant le projet GrandeBaleine. Voir BNQ, DANQ, 27 mars 1991, 7246.

90. Commission 1990, 31 mai, CET-2341. 
de commerce de la Rive-Sud ou l'Association des constructeurs de routes et grands travaux du Québec ${ }^{91}$.

Même si certains groupes se positionnent en faveur du nucléaire, comme l'Association des ingénieurs-conseils du Québec, l’École Polytechnique, le Groupe Lavalin ou la SGA internationale ${ }^{92}$, on sent que la ferveur nucléaire n'y est plus. En effet, outre la recherche sur la fusion nucléaire, le développement de l'énergie atomique ne semble plus faire partie des préoccupations des membres du PLQ. Quant aux élus du PQ, une décennie après l'établissement et le maintien du moratoire, ils n'ont pas vraiment changé d'opinion; ils s'opposent au projet Grande-Baleine sans toutefois préconiser un retour au développement de l'énergie nucléaire ${ }^{93}$.

À la lumière de ces prises de position, il semble que les représentations de l'énergie nucléaire se sont une fois de plus transformées: d'une image de l'énergie de l'avenir, souhaitée ou non, on passe au spectre de la production d'énergie nucléaire afin de mieux mettre l'accent sur la nécessité d'augmenter de la production d'hydroélectricité. Devenue un véritable repoussoir, l'énergie atomique est désormais synonyme de dangers et de risques réels sur la santé des individus et, surtout, sur l'environnement.

L'abandon de l'énergie nucléaire par les responsables politiques et les dirigeants d'Hydro-Québec peut être attribuée, comme nous l'avons vu, à différents facteurs d'ordre économique, politique et environnemental: augmentation des coûts des centrales nucléaires; problèmes environnementaux au sujet des risques d'accidents dans le contrôle des réacteurs et de l'entreposage des déchets radioactifs ${ }^{94}$; perception de l'hydroélectricité comme une énergie verte, propre, dans un contexte de lutte contre les gaz à effet de serre; etc. À ces facteurs s'ajoutent, dans les années 1990, celui de l'émergence du concept de «développement durable ${ }^{95}$ ». Ce fai-

91. Voir Commission 1990, 23 mai, CET-2010; Commission 1990, 29 mai, CET-2176.

92. Pour SGA internationale, en guise d'exemple, il faut mettre un terme aux grands projets hydroélectriques pour se concentrer sur une relance du nucléaire. Voir Réjean Aucoin, dans Commission 1990, 15 mai, CET-1735.

93. À l'ouverture de la commission parlementaire de 1993 qui étudie la situation et les perspectives de l'énergie électrique au Québec, ni Guy Chevrette (PQ) ni Lise Bacon (PLQ) n'abordent la question du nucléaire. Voir Lise Bacon et Guy Chevrette, dans BNQ, DANQ, Commission permanente de l'économie et du travail. Consultation générale sur la proposition du plan de développement 1993-1995 d'Hydro-Québec [ciaprès Commission 1993], 23 février 1993, 10 h40 pour la citation (voir de 10h10 à 10 h50 pour les autres informations).

94. À ce sujet, la catastrophe nucléaire de Tchernobyl (Ukraine), en 1986, contribue assurément à exacerber les représentations négatives de l'énergie nucléaire un peu partout dans le monde. Voir T. Price, Political Electricity..., op. cit., 290.

95. La notion de «développement durable» sous-entend la recherche d'un équilibre entre plusieurs éléments jugés complémentaires: le développement économique, l'utilisation respectueuse des ressources naturelles, la protection de l'environnement, l'habilitation citoyenne des populations locales, ainsi que 
sant, la ministre Lise Bacon, dans son allocution de fermeture de la commission parlementaire de 1993, met en lumière le lien qui existerait entre la production de l'hydroélectricité et les valeurs associées au développement durable ${ }^{96}$. Dans un nouveau contexte international de lutte contre les changements climatiques, il semble donc que le Québec et son hydroélectricité s'inscrivent dans la course pour une production "d'énergie verte» et "propre», une énergie qui puisse ainsi s'exporter, entre autres aux États-Unis où les autorités veulent fermer les nombreuses centrales au charbon.

L'intégration du nouveau principe de développement durable se voit progressivement dans les discours et valeurs des responsables politiques, des dirigeants d'Hydro-Québec et des différents groupes de pression qui interviennent au sujet des enjeux énergétiques. Les réflexions collectives entourant la Table de consultation du débat public sur l'énergie, présidée par Alban D’Amours, permettent de concrétiser ce phénomène qui, ultimement, mènera à la marginalisation du nucléaire. Mise sur pied après l'abandon du projet Grande-Baleine par le nouveau gouvernement péquiste de Jacques Parizeau dans le but de préparer le renouvellement de la politique énergétique québécoise, cette Table de consultation laisse la parole à plus de 285 intervenants pendant l'année $1995^{97}$. Comme le précise à juste titre le ministre François Gendron, le Québec peut "compter sur un certain nombre de consensus" avant même l'ouverture du débat public, notamment celui que «la société québécoise toute [sic] entière adhère aux objectifs fondamentaux du développement durable ${ }^{98}$.

Puisqu'il s'agit d'une consultation publique d'envergure dont un des principaux thèmes touche au "choix des filières énergétiques, avec un accent particulier sur les modes de production de l'électricité ${ }^{99}$ ", la question du nucléaire y est abordée. Du côté des défenseurs, notamment les Entreprises québécoises du secteur nucléaire et l’Association nucléaire

la mise en valeur d'une qualité de vie des habitants d'un territoire donné. Voir Ministère du Développement durable, Environnement et Parcs, «Le développement durable, repères historiques», www.md delcc.gouv.qc.ca/developpement/reperes.htm [dernière consultation le 9 décembre 2014].

96. En résumant son point de vue ainsi que celui de plusieurs intervenants entendus en commission, Lise Bacon affirme qu' “[é]conomiquement, écologiquement et socialement, l’hydroélectricité est, plus que jamais, une composante essentielle du développement durable de la planète». Voir Commission 1993, 25 mars, 17h10.

97. André Beauchamp, «La reconquête démocratique en environnement: une attente sacrée», L'Action nationale, 87, 7 (janvier 1997): 43-54.

98. BAN, François Gendron, Notes pour une allocution du ministre des Ressources naturelles monsieur François Gendron lors de la conférence de presse pour le lancement du Débat public sur l'énergie, Québec, le 7 février 1995, 4.

99. Ibid., 7 (voir aussi 6). 
canadienne, il s'agit désormais de présenter le nucléaire à la saveur du jour en lui accolant l'image d'une énergie verte qui contribue à la protection de l'environnement et au développement durable:

L'option nucléaire offre un des bilans environnementaux les plus intéressants. En effet, une centrale nucléaire utilise moins de territoire qu'une centrale hydroélectrique, éolienne ou solaire pour une production équivalente. L'impact sur les écosystèmes et sur les populations avoisinantes des centrales s'en trouve donc amenuisé. [...]

Enfin, les centrales nucléaires n'émettent aucun gaz à effet de serre, ni aucun gaz causant les pluies acides. Elles produisent très peu de déchets qui sont d'ailleurs déjà gérés de façon responsable depuis les débuts de l'industrie. C'est d'ailleurs un de leurs principaux avantages qui fait qu' elles contribuent positivement au développement durable ${ }^{100}$.

Le nucléaire représente ainsi un outil privilégié dans la lutte contre les changements climatiques, sujet de l'heure dans les années $1990^{101}$. Pour les opposants, dont STOP et les Ami-e-s de la Terre de Québec, il s'agit plutôt de reprendre les représentations négatives évoquées depuis la fin des années 1970 pour s'opposer à la construction de nouvelles centrales nucléaires au Québec - fondées notamment sur la production de déchets radioactifs dont la longévité se compte en milliers d'années ${ }^{102}$. Ce faisant, ils considèrent alors le nucléaire et le développement durable comme étant aux antipodes. Le Québec n'ayant aucune centrale électrique au charbon et une seule grande centrale au pétrole à cette époque, le nucléaire ne peut donc pas être perçu comme une solution de rechange à leurs yeux, surtout pas à l'égard de l'hydroélectricité qui représente déjà une énergie verte et renouvelable.

Si Hydro-Québec voit toujours l'hydraulique comme le principal moyen de production d'électricité, elle reconnaît néanmoins qu'elle devra développer certaines filières d'appoint, telle que la biomasse, l'énergie éolienne ou la cogénération au gaz naturel. Elle précise même qu' "[o]n pourrait ajouter à la liste les centrales nucléaires, si la population était prête à les

100. BAN, Entreprises québécoises du secteur nucléaire, L'énergie nucléaire: une option méconnue au Québec, août 1995, ii. Voir aussi BAN, Association nucléaire canadienne, L'avenir de l'énergie nucléaire au Québec, juillet 1995.

101. Ce discours est particulièrement présent dans les pays où le charbon est encore fortement utilisé pour la production d'électricité, comme les États-Unis. Voir Christian Stoffaës, «Développement durable : l'idéologie du siècle », Revue d'économie financière, 66 (2002): 296-297.

102. BAN, Les Ami-e-s de la Terre de Québec, Une régie indépendante qui veillerait aux besoins énergétiques du peuple québécois en évitant les impacts négatifs pour l'environnement et l'humanité, 11 août 1995, 41-42; BAN, STOP, Débat public sur l'énergie au Québec: phase de consultation, août 1995, 3-5. 
accepter et si leur coût diminuait ${ }^{103}$ ", reléguant cette filière énergétique au bas de la liste des priorités. Or, il semble que cette "accessibilité sociale», pour reprendre les termes d'Alban D'Amours, soit plutôt «faible». En effet, dans son rapport final Pour un Québec efficace publié en 1996, le président recommande «que la future politique énergétique soit fondée sur la satisfaction des besoins des citoyens par le développement durable ${ }^{104}$. En ce qui a trait au nucléaire, il souligne que plusieurs intervenants se positionnent contre cette forme d'énergie, certains demandant même le démantèlement de Gentilly- $2^{105}$. Il poursuit:

[i]l est évident que la filière nucléaire, fortement contestée dans la plupart des pays du monde, n'est pas la mieux placée pour répondre aux critères du développement durable, en raison notamment des risques liés aux déchets nucléaires, pour les générations futures ${ }^{106}$.

Nul doute que les recommandations du rapport D’Amours en matière d'énergie nucléaire ont été entendues, car la politique énergétique de 1996, qui laisse une large place au principe de développement durable, rend "improbable la construction de nouvelles centrales nucléaires». Au sujet de Gentilly-2, la nouvelle politique souligne néanmoins que celle-ci «doit continuer de fonctionner jusqu'à la fin de sa durée de vie utile», et ce, afin de permettre "au Québec de sauvegarder, dans une certaine mesure, ses acquis technologiques et industriels dans la filière ${ }^{107}$. Ce faisant, la nouvelle politique énergétique reconnaît toutefois de manière implicite qu'il pourrait y avoir fermeture au-delà «de sa durée de vie utile».

Ainsi, depuis le début des années 1990, le discours gouvernemental et hydro-québécois semble de nouveau marqué par un consensus: s'il faut préserver une expertise dans le domaine nucléaire, le Québec n’aura pas à construire d'autres centrales. En d'autres mots, le nucléaire n'est plus l'énergie de l'avenir. De même, grâce aux discours de nombreux groupes de pression, de même qu'à la montée du principe de développement durable, la table semble désormais mise pour un éventuel démantèlement

103. BAN, Hydro-Québec, Une politique énergétique pour le Québec du XXI' siècle: la vision d'HydroQuébec, 1995, 20.

104. BAN, Alban D’Amours (président), Pour un Québec efficace. Rapport de la Table de consultation du débat public sur l'énergie (Québec, Gouvernement du Québec - Ministère des Ressources naturelles, 1996), 1.

105. Voir notamment BAN, Regroupement pour la surveillance du nucléaire, Mémoire sur l'énergie nucléaire au Québec préparé par Gordon Edwards, Ph.D., président du RSN, sur l'occasion du Débat public sur l'énergie au Québec, [1995], 10-11.

106. BAN, Alban D’Amours (président), Pour un Québec efficace..., op. cit., 82.

107. Gouvernement du Québec - Ministère des Ressources naturelles, L'énergie au service du Québec: une perspective de développement durable (Québec, Gouvernement du Québec, 1996), 46. 
de Gentilly-2. Alors que la question de la fermeture est posée dans le rapport d'Alban D'Amours en $1996^{108}$, il semble qu'elle n'a toutefois pas été analysée sérieusement par Hydro-Québec et les responsables politiques avant que ne survienne le problème de la réfection de la centrale plus d'une décennie plus tard ${ }^{109}$.

Une analyse des conflits entourant l'énergie nucléaire permet au praticien de l'histoire de se montrer sensible aux valeurs et représentations véhiculées par les différents acteurs qui, prenant la parole dans le champ politique, tentent d'influencer la définition du bien commun québécois. Se déployant surtout sur une échelle relevant du macropolitique, ces débats politiques et conflits de représentations ont traversé différentes étapes entre le début des années 1960 et la fin des années 1990. D’un relatif consensus percevant le nucléaire comme l'énergie de l'avenir et le symbole d'une modernité technologique et économique, les responsables politiques et les dirigeants d'Hydro-Québec en sont venus à considérer cette forme d'énergie comme problématique, entre autres en raison de son inadéquation avec le concept de développement durable.

Il serait utile de mettre en lumière trois facteurs qui ont influencé les choix énergétiques, de même que l'expression de valeurs et représentations qui y sont associées. Présentes tout au long de la période étudiée, les prévisions de la demande orientent sans contredit les prises de position. De même, les aléas de la situation économique, en plus des perceptions que les acteurs ont de cette réalité économique et de ses conséquences dans le secteur énergétique, influencent cet exercice de planification. Ainsi, au nom des prévisions sur la croissance de la demande en électricité, les dirigeants d'Hydro-Québec décident de se tourner vers l'énergie nucléaire dans les années 1960 et 1970 et convainquent les responsables politiques du bien-fondé de cette démarche. Plus tard, en s'appuyant sur les prévisions à très long terme, la société d'État reste fermement convaincue, au moins jusqu’aux années 1990, de la nécessité de garder un

108. BAN, Alban D’Amours (président), Pour un Québec efficace..., op. cit., 82.

109. Lors des audiences publiques du BAPE de 2004 portant sur le projet de modification des installations de stockage des déchets radioactifs et réfection de Gentilly-2, les dirigeants d'Hydro-Québec annoncent qu'il serait peut-être préférable de rénover la centrale et d'allonger ainsi sa durée de vie pour des raisons d'approvisionnements en énergie. Voir É. Lyrette, Évolution des controverses..., op. cit., 78-79. Finalement annoncé par le gouvernement libéral de Jean Charest en 2008, le projet de réfection tergiverse toutefois pendant quelques années en raison de certains problèmes techniques et de l'augmentation astronomiques des coûts. Il sera finalement abandonné par le gouvernement Marois en 2012. 
pied dans l'industrie nucléaire. Cela permettrait de disposer de la compétence technique et scientifique nécessaire lorsque cette filière énergétique deviendra la seule source d'énergie possible et rentable à développer.

Un autre facteur concerne le contexte international et la circulation transnationale des représentations symboliques entourant le nucléaire, prenant bien entendu une coloration locale ou nationale en fonction du contexte énergétique et des préoccupations de la Cité. Ici, il s'agit surtout des représentations occidentales de l'énergie nucléaire comme source de progrès technologique et de modernité économique dans les années 1960 et 1970 - compris dans cet article comme étant le "positivisme technologique». Il s'agit aussi de la montée de l'écologisme et de l'environnementalisme dans les années 1970 et 1980 - parfois propulsée par des événements marquants comme l'accident nucléaire de Three Mile Island aux États-Unis ou de Tchernobyl en Ukraine. Ces représentations de l'énergie nucléaire influencent les prises de position des acteurs sociopolitiques québécois, et apportent parfois à leurs discours un poids plus important.

Enfin, la notion de conflits dans le champ politique met en lumière un dernier facteur essentiel: celui des différents rapports de force qui définissent les relations entre les acteurs étudiés. Les rares transformations de ces rapports de force, qui peuvent voir le jour dans le sillon d'une conjoncture particulière, expliquent en partie les changements apportés dans les prises de position des responsables politiques qui forment le gouvernement. C'est notamment le cas au milieu des années 1990, alors que les débats publics sur l'énergie permettent de constater l'importance qu’a prise la notion de développement durable au Québec, de même que son inadéquation avec le développement de l'énergie nucléaire. Avec l'étude de ces rapports de force, l'historien analyse alors la relation particulière entre l'habilitation citoyenne et l'exercice du pouvoir, entre le citoyen «engagé » et le responsable politique. 